\title{
The physical meaning of charge extraction by linearly increasing voltage transients from organic solar cells
}

Cite as: Appl. Phys. Lett. 103, 063904 (2013); https://doi.org/10.1063/1.4818267

Submitted: 03 May 2013 . Accepted: 24 July 2013. Published Online: 09 August 2013

Robert Hanfland, Martin A. Fischer, Wolfgang Brütting, Uli Würfel, and Roderick C. I. MacKenzie
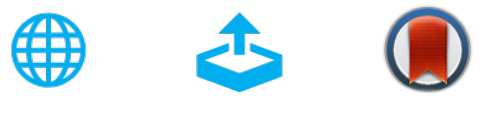

View Online

Export Citation

CrossMark

\section{ARTICLES YOU MAY BE INTERESTED IN}

Charge carrier extraction by linearly increasing voltage: Analytic framework and ambipolar transients

Journal of Applied Physics 108, 113705 (2010); https://doi.org/10.1063/1.3516392

The use of charge extraction by linearly increasing voltage in polar organic light-emitting diodes

Journal of Applied Physics 121, 175501 (2017); https://doi.org/10.1063/1.4982903

Injected charge extraction by linearly increasing voltage for bimolecular recombination studies in organic solar cells

Applied Physics Letters 101, 083306 (2012); https://doi.org/10.1063/1.4747330

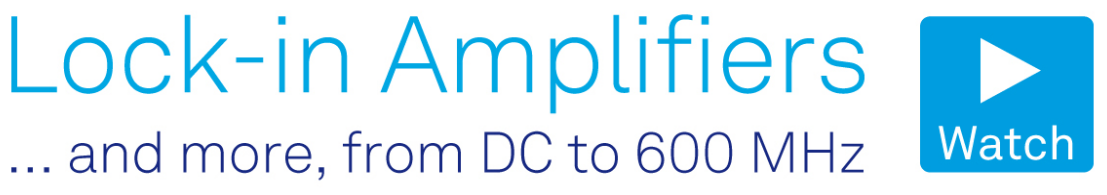




\title{
The physical meaning of charge extraction by linearly increasing voltage transients from organic solar cells
}

\author{
Robert Hanfland, ${ }^{1,2,3}$ Martin A. Fischer, ${ }^{1}$ Wolfgang Brütting, ${ }^{2}$ Uli Würfel, ${ }^{1,4}$ \\ and Roderick C. I. MacKenzie $3,5, a)$ \\ ${ }^{1}$ Fraunhofer Institute for Solar Energy Systems, Heidenhofstr. 2, 79110 Freiburg, Germany \\ ${ }^{2}$ Experimentalphysik IV, Institut für Physik, Universität Augsburg, Universitätsstr., 86159 Augsburg, Germany \\ ${ }^{3}$ FRIAS, University of Freiburg, Albertstr. 19, 79104 Freiburg, Germany \\ ${ }^{4}$ Material Research Center, University of Freiburg, Stefan-Meier-Str. 21, 79104 Freiburg, Germany \\ ${ }^{5}$ Faculty of Engineering, University of Nottingham, Nottingham NG7 2RD, United Kingdom
}

(Received 3 May 2013; accepted 24 July 2013; published online 9 August 2013)

\begin{abstract}
Carrier mobility in organic solar cells is almost exclusively determined with the Charge Extraction by Linearly Increasing Voltage (CELIV) technique; indeed much of our understanding of the recombination and charge transport mechanisms in organic solar cells is based on CELIV measurements. However, since the conception of the CELIV method, our understanding of organic semiconductors has significantly advanced. In this work, we critically examine the CELIV methods ability to provide accurate material data in the light of recent advances in our understanding of trap states and their influence on mobility in organic semiconductors. We then apply this knowledge to understand the mechanisms responsible for degradation in organic solar cells. (C) 2013 AIP Publishing LLC. [http://dx.doi.org/10.1063/1.4818267]
\end{abstract}

Organic solar cells offer the potential of a low-cost, ${ }^{1}$ low-carbon source of electricity. Within the last five years, power conversion efficiencies have increased from $3 \%^{2}$ to over $10 \%^{3}$ today. However, to further increase energy conversion efficiencies, a larger proportion of the photogenerated charge carriers need to reach the contacts of the cell. ${ }^{4}$ To achieve this, the lifetime $(\tau)$ and the mobility $(\mu)$ of the materials charge carriers must be maximized. ${ }^{4}$ Researchers often use the Charge Extraction by Linearly Increasing Voltage (CELIV) $)^{5,6}$ method to measure mobility ${ }^{7,8}$ within research devices to guide both device and material development. However, since Juska ${ }^{5,6}$ first pioneered the CELIV method, our understanding of organic semiconductors has considerably improved..$^{9,10}$ In particular, our understanding of charge transport in these material systems has considerably developed. ${ }^{11}$ In this letter, we examine the underlying theoretical assumptions made by $\mathrm{Juska}^{6}$ in the derivation of the CELIV measurement technique and hold them up to scrutiny against today's knowledge of organic semiconductor materials. ${ }^{9}$ The ability to accurately measure mobility in organic semiconductors is essential if the material systems used in organic solar cells are to continue to be developed.

In Juska's ${ }^{5}$ original paper, he derived the now well known equation to extract mobility from CELIV transients,

$$
\mu=\frac{2}{3} \frac{d^{2}}{A t_{\max }^{2}\left(1+0.36 \frac{\Delta j}{j(0)}\right)},
$$

where, $A$ describes the ramp rate $(V / s)$ of a negative triangular voltage function applied to the cell; this voltage ramp usually starts at around $0 \mathrm{~V}$ and decreases to typically $-1 \mathrm{~V}$ to $-5 \mathrm{~V}$ of applied bias within $10-20 \mu s ; d$ is the thickness of the device; $\Delta j / j(0)$ is the ratio of the current due to charge

\footnotetext{
a)roderick.mackenzie@nottingham.ac.uk
}

being extracted from the organic semiconductor divided by the current due to the geometric capacitance of the device; and $t_{\max }$ is the time at which the maximum current is observed in the transient. A typical CELIV transient is depicted in Figure 1. In the derivation of Eq. (1), Juska described the most mobile carrier species as a uniform sheet of charge of carrier density $\mathrm{n}\left(\mathrm{m}^{-3}\right)$, which extends across the device. As the voltage ramp is applied, Juska describes this charge sheet being uniformly swept to the extracting contact leaving a region of length $l(x)$, depleted of charge behind it. Juska assumed band like transport, neglecting the presence of traps. Today, however, it is well known that up to $90 \%$ of charge in a working organic solar cells resides in deep trap states; ${ }^{12,13}$ and the deeper the trap the longer it will take for a carrier to be released. Thus, rather than a well defined sheet of charge being swept out of the device, it is far more likely that the charge sheet will become spread out and distorted by carrier trapping and escape events. Therefore, it may not be possible to neglect carriers traps in the derivation of the CELIV method. Furthermore, Juska assumed that the material had one constant free carrier mobility. However, today it is well known that the mobility is a strong function of carrier density and in turn carrier density is known to be a strong function of applied voltage ${ }^{14}$ thus the mobility of the material will change as the CELIV voltage ramp is applied to the device to extract the carriers. The relationship between mobility within a device and potential applied to it can be described by the equation,

$$
\mu_{e / h}=\frac{1}{d} \int_{0}^{d} \mu_{e / h}^{0} \frac{n_{e / h}^{\text {free }}(x, V)}{n_{e / h}^{\text {free }}(x, V)+n_{e / h}^{\text {trapped }}(x, V)} d x,
$$

where $\mu_{e / h}^{0}$ is the mobility of free carriers, $n_{e / h}^{\text {free }}$ is the density of free carriers, $n_{e / h}^{\text {trapped }}$ is the density of trapped carriers, $V$ is the applied voltage, and d is the device thickness; this equation explicitly states that the mobility of trapped carriers is 


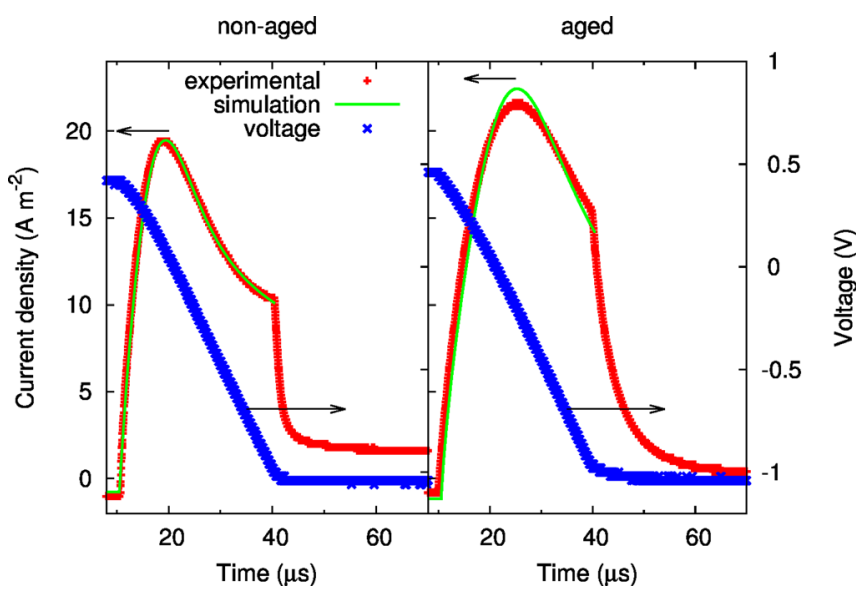

FIG. 1. Experimental CELIV measurements (a) a non-aged cell and (b) a cell aged for $1176 \mathrm{~h}$ using a UV source equivalent to exposure at $1 \mathrm{Sun}$ at $45^{\circ} \mathrm{C}$ with a relative humidity of $6 \%$.

zero. Thus, we must ask if the mobility of the material in the device is changing during the measurement, how does this effect our value of mobility as measured by the CELIV method?

Finally, the CELIV method was derived for a material system where one material had a very low mobility and the other carrier species had a high mobility. However, due to improved polymers, ${ }^{15}$ today's high efficiency devices have more balanced mobilities and it is thus not clear that one carrier remains immobile and thus CELIV theory holds. In the following pages, we address these questions using a combination of experimentation and theory; the result is a better understanding of how CELIV transients can be interpreted.

Inverted bulk-heterojunction devices were fabricated with a $\mathrm{Cr} / \mathrm{Al} / \mathrm{Cr} / \mathrm{P} 3 \mathrm{HT}: \mathrm{PCBM} / \mathrm{PEDOT}: \mathrm{PSS} / \mathrm{Au}$-grid structure. $^{16}$ Poly(3-hexylthiophene-2,5-diyl) (P3HT) and phenyl-C61butyric acid methyl ester (PCBM) were mixed in a weight ratio of 1:0.67. The active and poly(3,4-ethylenedioxythiophene) poly(styrenesulfonate) (PEDOT:PSS) $(90 \mathrm{~nm})$ layer were deposited by spin coating. The metal grid was thermally evaporated. Devices were annealed at $130{ }^{\circ} \mathrm{C}$ for $10 \mathrm{~min}$. Cell aging was performed using a UV source equivalent to exposure under AM $1.5 \mathrm{G}$ radiation at $45^{\circ} \mathrm{C}$ with a relative humidity of $6 \%$ for $1176 \mathrm{~h}$. The resulting CELIV transients before and after aging are plotted in Figure 1. By applying Eq. (1) to the experimental data, a mobility of $9.5 \times 10^{-9} \mathrm{~m}^{2} \mathrm{~V}^{-1} \mathrm{~s}^{-1}$ before the cell is aged is obtained, and a decreased mobility of $2.3 \times 10^{-9} \mathrm{~m}^{2} \mathrm{~V}^{-1} \mathrm{~s}^{-1}$ after aging is obtained.

Before attempting to understand the change in measured mobility upon degradation, we first use our device model ${ }^{17}$ to better understand the physical mechanisms which can alter CELIV transients. To model CELIV transients from an organic solar cell, we use an effective medium approximation where the LUMO level of the fullerene is taken as the electron mobility edge and the HOMO level of the polymer is taken as the hole mobility edge. To calculate the electric field profile within the device, Poisson's equation is solved in one dimensional,

$$
\frac{d}{d x} \cdot \epsilon_{0} \epsilon_{r} \frac{d \phi}{d x}=q\left(n_{t o t}-p_{t o t}\right)
$$

where $\epsilon_{0}$ is the permittivity of free space, $\epsilon_{r}$ is the relative permittivity of the medium, $n_{t o t}\left(p_{t o t}\right)$ is the sum of the free and trapped electron (hole) population. To simulate the movement of free carriers, the bi-polar drift diffusion equations are solved,

$$
\begin{aligned}
& \boldsymbol{J}_{\boldsymbol{n}}=q \mu_{e} n \frac{\partial E_{L U M O}}{\partial x}+q D_{n} \frac{\partial n}{\partial x} \\
& \boldsymbol{J}_{\boldsymbol{p}}=q \mu_{h} p \frac{\partial E_{H O M O}}{\partial x}-q D_{p} \frac{\partial p}{\partial x}
\end{aligned}
$$

where $\mu_{e}\left(\mu_{h}\right)$ is the free electron (hole) mobility, $E_{L U M O}$ $\left(E_{\text {HOMO }}\right)$ is the spatially dependent potential of the LUMO (HOMO), $D_{n}\left(D_{p}\right)$ are the electron (hole) diffusion coefficients. Conservation of particles is forced using the carrier conservation equations and to describe carrier trapping and recombination we use the Shockley-Read-Hall model. ${ }^{18}$ The carrier traps are defined as an exponential distribution of states,

$$
\rho^{e / h}(E)=N^{e / h} \exp \left(-E / E_{u}^{e / h}\right),
$$

where $N^{e / h}$ are the electron/hole trap densities at the LUMO and HOMO edge; $E_{u}^{e / h}$ are the characteristic electron/hole tail slope energies and $E$ is the distance from the LUMO/HOMO edge. A full list of device parameters are given in the supplementary information, ${ }^{19}$ parameters were chosen to be symmetric and to be close to those already reported in the literature. ${ }^{9}$

If Eq. (1) is a measure of free carrier mobility as described by Juska in his derivation, then the density of carrier traps should not affect the shape of the CELIV transient. To test Juska's approximation, Figure 2 plots five simulated CELIV transients with all device parameters held constant except for the carrier trap densities. It can be seen that an increase in the density of carrier trap states not only increases the magnitude of the CELIV peak, but also shifts the CELIV peak to the right. If one uses Eq. (1) to extract the mobilities, we obtain values of $1.1 \times 10^{-7}$ to $3.0 \times 10^{-9} \mathrm{~m}^{2} \mathrm{~V}^{-1} \mathrm{~s}^{-1}$. Thus, we can determine from this

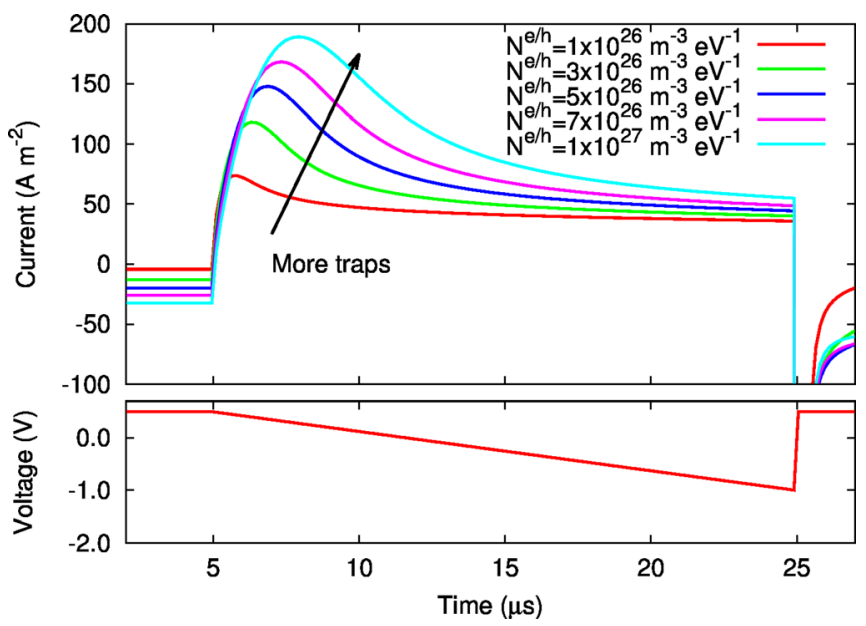

FIG. 2. The influence of carrier trap states on the CELIV transient. It can be seen that the density of trap states can shift the position and magnitude of the transient. The CELIV method gives the mobility of the curve with a trap density of $1 \times 10^{26} \mathrm{~m}^{-3}$ as $1.1 \times 10^{-7} \mathrm{~m}^{2} \mathrm{~V}^{-1} \mathrm{~s}^{-1}$, and the mobility of curve with a trap density of $1 \times 10^{27} \mathrm{~m}^{-3}$ as $3.0 \times 10^{-9} \mathrm{~m}^{2} \mathrm{~V}^{-1} \mathrm{~s}^{-1}$. 
graph that CELIV is measuring an averaged carrier mobility (as described by Eq. (2)), which includes the influence of carrier trap states.

As was discussed above, carrier mobility in organic semiconductors is known to change as a function of applied bias and as the CELIV technique intrinsically uses a voltage ramp to extract charges from the device. An obvious question to ask is how much does the application of a voltage ramp to the cell affects the measured mobility. Figure 3 plots the average carrier mobility within the device as a function of time as calculated using Eq. (2). It can be seen that the mobility within the device changes by up to an order of magnitude within the CELIV transient and by up to $50 \%$ before the peak of the transient.

If the trap density and applied voltage both affect the CELIV transient, then we should ask how good a measurement of mobility is the CELIV method in general? To answer this question, a series of CELIV simulations were performed where the free electron and hole mobilities $\left(\mu_{e / h_{8}^{0}}^{0}\right.$ were set equal and varied together from $\mu_{e}^{0}=\mu_{h}^{0}=1 \times 10^{-8}$ to $1 \times 10^{-4} \mathrm{~m}^{2} \mathrm{~V}^{-1} \mathrm{~s}^{-1}$. Equation (1) was used to extract the mobilities from the CELIV transients and Eq. (2) used to extract the effective mobility from the model before the CELIV transient started. This was repeated for energetic tail slope energies of $5 \mathrm{meV}, 25 \mathrm{meV}, 50 \mathrm{meV}$, and $100 \mathrm{meV}$. Figure 4 plots the results. The black line is a guide to the eyes representing the case where CELIV would measure exactly the effective device mobility as given by Eq. (2). It can be seen that for a very shallow tail slope $(5 \mathrm{meV})$, the mobility as extracted from the CELIV transient is within an order of magnitude of the average device mobility. As more disorder is introduced into the device by increasing the tail slope energy, it can be seen that the CELIV method becomes less accurate. This is because the CELIV derivation assumes that charge is extracted from the device as a single well defined charge sheet moving from one contact to the other leaving a region of width $l(x)$ depleted of charge behind it (see left hand side of Figure 5). With the addition of disorder into the device, carriers in shallow traps can become extracted from the device faster than carriers in deeper traps

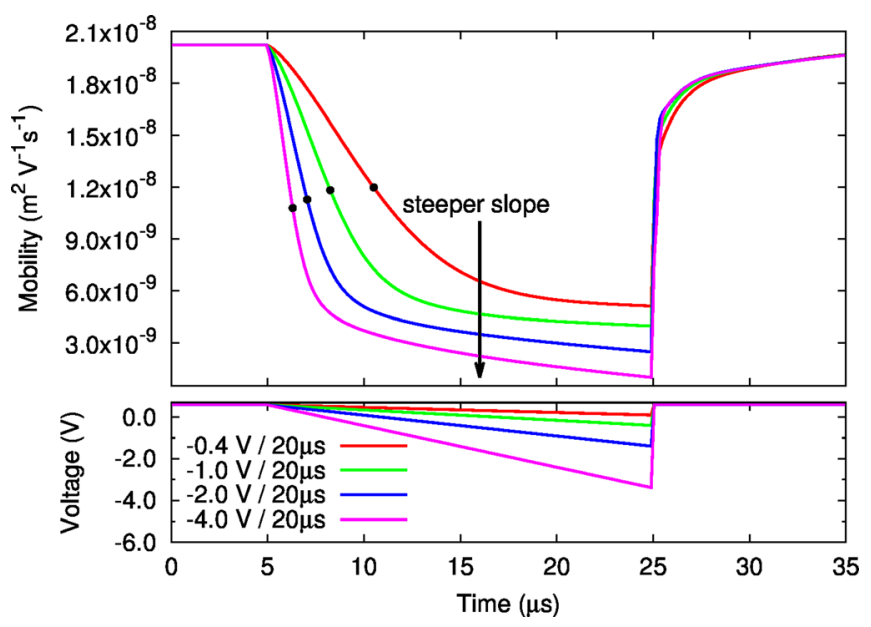

FIG. 3. The influence of the voltage ramp rate on the average mobility within the device as calculated with Eq. (2). The black dots show where the peak of the CELIV transient occurs, it can be seen that the measurement process its self can change the mobility by up to $50 \%$ before the CELIV peak.

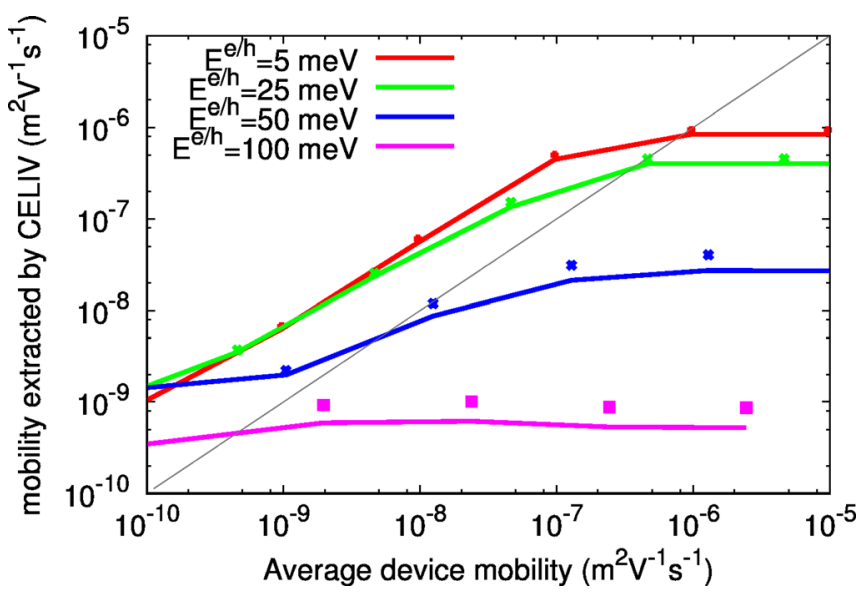

FIG. 4. Device mobility calculated with Eq. (2) plotted against the mobility extracted from the CELIV transients for different levels of disorder. Extracted mobility was calculated after Deibel ${ }^{20}$ (dots) and Juska (lines). It can be seen that CELIV can estimate mobility to within an order of magnitude for a device with a low amount of disorder.

(see right hand side of Figure 5); this is because during the application of the CELIV voltage ramp the quasi-Fermi lev$\mathrm{el}(\mathrm{s})$ of the free carriers will progressively move to lower energies forcing ever more deeply trapped states to release their carriers in order to move towards equilibrium, furthermore shallowly trapped carriers take less time to thermalize and become mobile than carriers in deeper traps. Thus rather than a single uniform charge sheet being removed from the device, charge is removed progressively from deeper and deeper traps across the entire device. This means that the charge sheet (and region $l(x)$ ) in the CELIV derivation
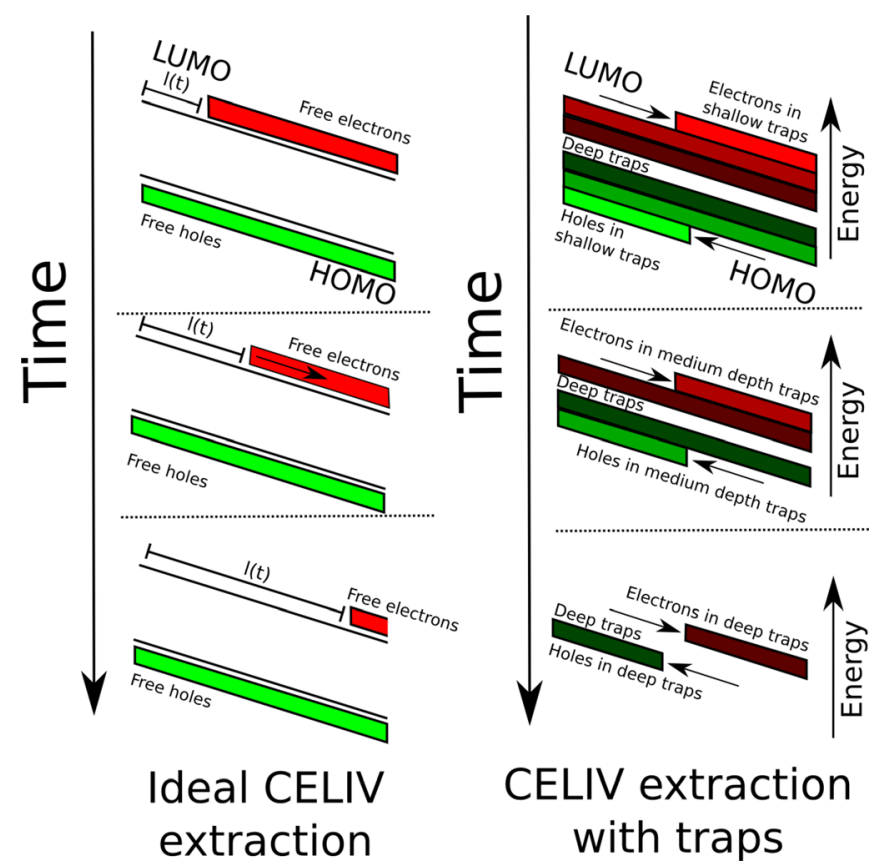

FIG. 5. Schematic diagram comparing the idealized removal of carriers from a device with no traps as described in the derivation of the CELIV method, and how carriers are removed from a device when traps are present. In the device with traps, charge carriers are removed progressively from deeper and deeper traps across the entire device. Thus the distance $l(x)$ defined in CELIV theory becomes poorly defined. Also, CELIV assumes only one mobile charge carrier (left) while high efficiency solar cells have more balanced mobilities (right). 
becomes ill defined and CELIV becomes less accurate. Organic solar cells often have mobilities ranging from $1 \times 10^{-9}$ to $1 \times 10^{-6} \mathrm{~m}^{2} \mathrm{~V}^{-1} \mathrm{~s}^{-1}$ and tail slopes from $30 \mathrm{meV}$ to $70 \mathrm{meV}$, thus we would expect CELIV to be accurate to within two orders of magnitude. See the supplementary information for a more detailed analysis of this process. ${ }^{19}$ Also included in the supplementary information are investigations into how asymmetric mobilities affect CELIV transients. We find that CELIV tends to measure the mobility of the most mobile charge carrier and asymmetric mobilities do not cause a double peak in the measured current.

From the above discussion, it is clear that both free carrier mobility and the density/distribution of trap states are key parameters in defining the shape of CELIV transients. This brings us back to the question of how to understand the degradation data in Figures 1(a) and 1(b). To understand what the aging process is physically doing to our cell, the numerical model was fit simultaneously to the CELIV transient from the non-degraded cell in Figure 1(a) and the light and dark JV curves. To perform the fit, mobility, trap densities, tail slope energies were altered. ${ }^{19}$ After the model was calibrated, we were able to fit the aged experimental data by only further adjusting the carrier trap densities, we can therefore say during the aging process additional trap states are generated within the material (possibly due to the introduction of water and oxygen) and although CELIV measurements could be interpreted to suggest the free carrier mobility is being reduced this is not necessarily the case.

In conclusion, we have applied a modern model and understanding of organic semiconductors in combination with experiments to evaluate a method of experimentally measuring mobility originally proposed by Juska. We concluded that; (a) carrier trap states change the shape of the CELIV transient significantly; (b) the CELIV measurement itself changes the average carrier mobility by up to $50 \%$; (c) the mobility as measured by CELIV can provide a good estimate to the mobility of the most mobile charge carrier for ordered materials, however, for materials with a high density of trap states, the estimate may be less reliable. For typical organic solar cells the accuracy of CELIV is within one or two orders of magnitude. We demonstrate that the change in the CELIV transient upon aging can be explained by the formation of trap states.

We are grateful for access to the University of Nottingham High Performance Computing Facility.

${ }^{1}$ C. J. Emmott, A. Urbina, and J. Nelson, Sol. Energy Mater. Sol. Cells 97, 14-21 (2012).

${ }^{2}$ M. A. Green, K. Emery, Y. Hishikawa, and W. Warta, Prog. Photovoltaics 15, 425-430 (2007).

${ }^{3}$ M. A. Green, K. Emery, Y. Hishikawa, W. Warta, and E. D. Dunlop, Prog. Photovoltaics 20(1), 12-20 (2012).

${ }^{4}$ H. Azimi, A. Senes, M. C. Scharber, K. Hingerl, and C. J. Brabec, Adv. Energy Mater. 1(6), 1162-1168 (2011).

${ }^{5}$ G. Juška, K. Arlauskas, M. Viliūnas, and J. Kočka, Phys. Rev. Lett. 84, 4946-4949 (2000).

${ }^{6}$ G. Juška, K. Arlauskas, M. Viliūnas, K. Genevičius, R. Österbacka, and H. Stubb, Phys. Rev. B 62, R16235-R16238 (2000).

${ }^{7}$ G. F. Seynhaeve, R. P. Barclay, G. J. Adriaenssens, and J. M. Marshall, Phys. Rev. B 39(14), 10196-10205 (1989).

${ }^{8}$ J. Kniepert, M. Schubert, J. C. Blakesley, and D. Neher, J. Phys. Chem. Lett. 2(7), 700-705 (2011).

${ }^{9}$ T. Kirchartz, B. E. Pieters, J. Kirkpatrick, U. Rau, and J. Nelson, Phys. Rev. B 83(11), 115209 (2011).

${ }^{10}$ G. Garcia-Belmonte and J. Bisquert, Appl. Phys. Lett. 96(11), 113301 (2010).

${ }^{11}$ A. Foertig, J. Rauh, V. Dyakonov, and C. Deibel, Phys. Rev. B 86, 115302 (2012).

${ }^{12}$ R. C. I. MacKenzie, T. Kirchartz, G. F. A. Dibb, and J. Nelson, J. Phys. Chem. C 115(19), 9806-9813 (2011).

${ }^{13}$ Y. Roichman and N. Tessler, Appl. Phys. Lett. 80(11), 1948-1950 (2002).

${ }^{14}$ C. G. Shuttle, R. Hamilton, J. Nelson, B. C. O'Regan, and J. R. Durrant, Adv. Funct. Mater. 20(5), 698-702 (2010).

${ }^{15}$ B. K. Yap, R. Xia, M. Campoy-Quiles, P. N. Stavrinou, and D. D. Bradley, Nature Mater. 7(5), 376-380 (2008).

${ }^{16}$ B. Zimmermann, U. Würfel, and M. Niggemann, "Processing and preparation of polymer and organic solar cells," Sol. Energy Mater. Sol. Cells 93(4), 491-496 (2009).

${ }^{17}$ R. C. I. MacKenzie, C. G. Shuttle, M. L. Chabinyc, and J. Nelson, Adv. Energy Mater. 2, 662-669 (2012).

${ }^{18}$ R. C. I. MacKenzie, C. G. Shuttle, G. F. Dibb, N. Treat, E. von Hauff, M. J. Robb, C. J. Hawker, M. L. Chabinyc, and J. Nelson, J. Phys. Chem. C 117(24), 12407-12414 (2013).

${ }^{19}$ See supplementary material at http://dx.doi.org/10.1063/1.4818267 for detailed simulation results.

${ }^{20}$ C. Deibel, Phys. Status Solidi A 206(12), 2731-2736 (2009). 